\title{
Cryotherapy in Dendritic Keratitis.
}

\author{
C.D. MPYET and RON. GHOSH DO
}

From: University of Jos Teaching Hospital, Jos, Nigeria.

\section{SUMMARY:}

This study evaluates the effectiveness of cryotherapy in the treatment of dendritic Keratitis where antiviral agents are not available. The results show some improvement in visual acuity while one patient has a drop in vision. The extent of corneal scarring appears to depend on the duration of the disease and extent of stroma involvement. A comparative study with antiviral agents is recommended. To evaluate the effectiveness of cryotherapy in the treatment of dendritic keratitis where antiviral agents are not available. Ten patients with dendritic keratitis underwent cryotherapy by a freeze thaw technique at a temperature of between $-40^{\circ} \mathrm{C}$ to $-60^{\circ} \mathrm{C}$. Six patient records were reviewed while the remaining four were prospectively followed up. Patients had Gutt atropine sulphate $1.0 \%$ and Oc. Chloramphenicol $1.0 \%$ in addition to their treatment. There was an improvement in the visual outcome of most patients with complete epithelial healing within ten days of the procedure. We recommend a comparative study with antiviral agents though cryotherapy can be considered where antiviral agents are not available.

KEY WORDS: Dendritic keratitis, cryotherapy, antiviral agents.

\section{INTRODUCTION}

Herpes simplex keratitis is a common cause of corneal disease leading to corneal scarring or perforation and permanent impairment or loss of vision worldwide. It is thought to be one of the most common causes of ocular disease in the United States, being second only to trauma as a cause of corneal blindness ${ }^{1}$. In a developing country like Nigeria, exact figures are not readily available but this disease may not be uncommon especially with the advent of Human immunodeficiency virus infection.

Herpes simplex virus type I is usually involved although serotype II is occasionally implicated in dendritic keratitis. Herpetic comeal ulcers may appear anywhere in the cornea. They are characteristically thin and branched. This ulcer may enlarge and deepen to become an amoeboid ulcer. These ulcers are usually found in the epithelium and superficial layers of the corneal stroma. The heal spontaneously in about two to four weeks ${ }^{2}$. An ulcer involving the stroma will leave a residual scar but an ulcer confined to the epithelium heals without scarring.

Various complications may follow herpetic infections ${ }^{2}$. These include, corneal anaesthesia recurrences, stromal keratitis, iritis with secondary glaucoma and indolent ulcers.

The primary ocular infection by Herpe simplex virus is mild, without significant symptom and may not require treatment ${ }^{2}$. The virus then remains dormant in the spinal nerve ganglia until months or years later when secondary infection occurs. The secondary infection usually appears as localized epithelial ulcers and recur at irregular intervals, this requires treatment as it is usually symptomatic and can last for up to three weeks.

Currently, the most effective method of treating herpetic ulcers is by the use of antiviral drugs. These are not readily availabie in this part of the world and where available, are not affordable. Our experience here has shown that patients resort to use of traditional medications or nothing at all when they cannot afford these drugs. Thus, a primary epithelial disease would have extended to the deep stroma or sometimes complicated by endophthalmitis. Other options for the treatment of dendritic keratitis were therefore considered. Non-antiviral techniques mentioned in the literature for the treatment of dendritic keratitis include; mechanical 
debridement ${ }^{1,2,3,4}$ and chemical cauterization using phenol, alcohol or iodine ${ }^{2}$. Topical cyclosprinA has been used in the treatment of stromal herpes simplex keratitis ${ }^{5}$. Very little is mentioned in literature about cryotherapy and its outcome even though it has been described in the ophthalmic literature for about forty years ${ }^{4}$. A combined retrospective and prospectives study to look at the outcome of cryotherapy in dendritic ulceration over a period of ten years was undertaken.

\section{PATIENTS AND METHODS}

Case records of six patients with dendritic ulceration of the cornea who had undergone this procedure were looked into. Four other patients were prospectively followed up. At diagnosis, patients were started on Guttae Atropine Sulphate 1.0\% three times daily in the affected eye and were given appointment for the procedure, after the procedure has been explained to each patient and an informed
The retinal probe of the cryoamoils machine was applied to the affected area. The diseased tissue and adjacent healthy cornea were treated, by activating the probe through the foot pedal, at a temperature of between $-40 \mathrm{C}$ to $-60 \mathrm{C}$ over a period of ten seconds at each site of application. The iceball was disrupted by switching off the flow of gas to the probe through the foot pedal of the cryomachine. Oc. Chloramphenicol $1.0 \%$ was applied and the eye was padded. Patients were reviewed after twenty-four (24) hours when the pad was removed and were continued on Guttae atropine $1.0 \%$ tide, Oc chloramphenicol $1.0 \% \mathrm{~b} . \mathrm{d}$ patients were then reviewed at least every two days until healing was completed and thereafter given longer appointments.

\section{RESULTS}

There were 10 patients of whom 6 were male and 4 were female. The summary of the clinical data and nature of treatment is shown in the table below:

Table 1

\begin{tabular}{lrlcccc}
\hline Serial No. & Age & Sex & Duration of disease & Visual acuity & Time for epithelial healing & Visual outcome \\
\hline 1. & 47 & M & $3 / 52$ & $6 / 18$ & $6 / 7$ & $6 / 12$ \\
2. & 17 & F & $4 / 52$ & $6 / 18$ & $7 / 7$ & $6 / 9$ \\
3. & 38 & M & $4 / 52$ & $6 / 36$ & $7 / 7$ & $6 / 60$ \\
4. & 8 & M & $4 / 52$ & PL & $5 / 7$ & $6 / 36$ \\
5. & 50 & F & $5 / 52$ & $6 / 24$ & $7 / 7$ & $6 / 24$ \\
6. & 6 & F & $1 / 52$ & N.R & $5 / 7$ & LTF \\
7. & 29 & M & $3 / 52$ & CF & $7 / 7$ & $6 / 12$ \\
8. & 10 & M & $2 / 52$ & $6 / 12$ & $7 / 7$ & LTF \\
9. & 21 & F & $4 / 52$ & $6 / 12$ & $9 / 7$ & $6 / 9$ \\
10. & 16 & M & $2 / 52$ & CF & $10 / 7$ & $6 / 24$ \\
\hline
\end{tabular}

NR - Not recorded

LTF $\quad-$ lost to follow-up

consent obtained.

The affected eyes were anaesthesized with loca anaesthetic (1\% tetracaine) and the cornea epithelium was stained with fluorescene highlight the ulcerated area in green. The lids were held open with a lid speculum.
In most patients epithelial healing was completed after seven days of treatment. In patients who had the disease for a longer period of time before cryotherapy, stromal infiltration was usually denser after healing. Four patients had corneal macula after healing while four others had corneal leucoma. 
One patient showed a drop in visual acuity by one line. In another patient, there was no change in visual acuity. Two others were lost to follow-up. The remaining six patients showed an improvement in vision after treatment. The final visual acuity after healing was affected by the location of the ulcer. Patients with peripherally located ulcers had better visual acuities.

\section{DISCUSSION}

The cryoprobe forms a small iceball on the cornea, this causes frosting of the epithelial cells and their contents. Thawing causes disruption of the iceball. This results in distuption and destruction of the cell and its contents. The diseased cells are then replaced by healthy epithelium growing over the area. The local anaesthetic is usually adequate and this procedure is accepted by most patients as painless. In most cases, healing is complete by the end of one week without residual corneal staining and most patients do not complain of any when they come for follow-up after the first week. This is a similar healing period as reported with the use of acyclovir, triflurothymidine adenine arabinoside $e^{6,7}$ and ganciclovir gel ${ }^{8}$.

Toxic effects of antiviral antimetabolites have been reported ${ }^{9}$. With cryotherapy, these are circumvented. We have not noted corneal side effects with cryotherapy in dendritic keratitis.

While one patient showed a drop in visual acuity, most others had an improvement in visual acuity. The most important factor here is the location of the ulcer and therefore the resultant scar. The resultant corneal opacity seems to depend on the extent of stromal involvement as suggested by other authors ${ }^{10}$. The cost of cryotherapy in our centre is just about a quarter of the cost of antiviral agents. This, many patients can afford and with healing occurring within seven days of treatment, patients are unlikely to seek other methods of treatment. This circumvents the alternative use of traditional medications and the complications that could flow.

\section{CONCLUSION}

This study is an open non-comparative study.

It would be advisable to compare the outcome of cryotherapy with antiviral therapy before strong recommendations could be made. However, in dendritic keratitis, where antiviral agents are either not available or affordable, cryotherapy, where available, could be an alternative method of treatment with reasonably good results.

\section{REFERENCES}

1. Basic and clinical sciences course, section 8 , external disease and cornea. American Academy Ophthalmology, San Francisco, California 1991. p108-112.

2. John Sandforth-smith: Eye diseases in hot climates 2nd edition. Butterworth Heinemann Ltd. 1990: p. 95-96.

3. Helveston EM, Ellis FD. Paediatric Ophthalmology Practice CV. Mosby Company 1980. p 242.

4. Catford GV. Cryotherapy in Ophthalmology in Miller SJ. (ed), Operative surgery. Butterworth (Publishers) Ltd, London 1976. p 142-145.

5. Heilingenhaus A, Steuhl KP. Treatment of HSV-1 stromal keratitis with topical cyclosporinA: a pilot study. Graefes Archives for Clinical and Experimental, Ophthalmology. 1999, 237(5): 4358.

6. Lalau C, Oosterphuis JA, Versteeg J, VanRij G, Renardel De Laralette JGC, CrGandijkA and Lamers WPMA. Acyclovir and Triflorothymidine in herpetic keratitis: A multicentre trial, $\mathrm{Br}$. J. Ophthalmol. 1982; 66: 506-508.

7. McGill J, Tormey P, Walker CB. Comparative Trial of acyclovir and adenine arabinoside in the treatment of herpes simplex corneal ulcers. Br. J. Ophthalmol, 1981; 65: 610-613.

8. Colin J, Hoh HB, Easty dL, Herbort CP, ReskniKoff S, Riyal D, Romdane K. Ganciclovir ophthalmic gel (Virgan $0.15 \%$ ) in the treatment of herpes simplex Keratitis. Cornea. 1997, 16(4): 393-9.

9. Ohashi Y. Treatment of herpetic keratitis with acyclovir: benefits and problems. Ophthalmological. 1997, 211 suppl 1: 29-32.

10. Schwab L. Eye Care in Developing Nation 3rd Edition. The Foundation of the American Academy of Ophthalmology 1999: p 135-136. 